\title{
A RELAÇÃO ENTRE ESTADO NUTRICIONAL E PRESENÇA DE DOENÇAS CRÔNICAS E SEU IMPACTO NA QUALIDADE DE VIDA DE IDOSOS: REVISÃO INTEGRATIVA
}

\author{
THE RELATIONSHIP BETWEEN NUTRITIONAL STATE AND PRESENCE OF CHRONIC DISEASES \\ AND ITS IMPACT ON THE QUALITY OF ELDERLY LIFE: INTEGRATIVE REVIEW
}

\author{
Sonia Lira ${ }^{a}$, Rita Monteiro Goulart ${ }^{b}$, Angélica Castilho Alonso ${ }^{c}$ \\ alira-sonia@hotmail.com, brtiagoulartnutri@gmail.com, cangelicacastilho@msn.com, \\ Universidade São Judas Tadeu - São Paulo (SP), Brasil
}

Data de recebimento do artigo: 23/02/2017

Data de aceite do artigo: 28/06/2017

\section{RESUMO}

Objetivo: Realizar uma revisão integrativa com objetivo de avaliar a relação do estado nutricional e da presença de doenças crônicas não transmissíveis com a qualidade de vida de idosos. Método: Procedeuse uma busca dos termos que definissem estado nutricional, doenças crônicas e qualidade de vida nos DeCS. Em seguida, realizou-se uma revisão da literatura nas bases de dados PubMed, SciELO, LILACS e Scopus. Foram incluídos estudos em português e em inglês, publicados no período de 2009 a 2016, realizados com seres humanos, disponíveis completos e com tema compatível ao pesquisado. Resultados: Foram utilizados instrumentos específicos para avaliação da qualidade de vida, estado nutricional e estado de saúde. A alteração do estado nutricional e a presença de doenças crônicas contribuíram para maior comprometimento da qualidade de vida no processo de envelhecimento. Conclusóes: A maior parte dos artigos evidenciou a associação entre estado nutricional e presença de doenças crônicas, o que compromete significativamente a qualidade de vida dos idosos.

Palavras-chave: Estado nutricional; doença crônica; qualidade de vida; meia-idade; idoso.

\begin{abstract}
Objective: To carry out an integrative review with the objective of evaluating the relationship between nutritional status and presence of chronic non-communicable diseases and the quality of life of the elderly. Method: A search was made for the terms that defined nutritional status, chronic diseases and quality of life in the Decs. Afterwards, a review of the literature was performed in PubMed, SciELO, LILACS and Scopus databases. We included studies in Portuguese and English, published from 2009 to 2016, carried out on human beings, available in full version and compatible themes. Results: Specific instruments were used to evaluate quality of life, nutritional status and health status. The change in nutritional status and the presence of chronic diseases lead to a greater impairment of the quality of life in the aging process. Conclusions: Most of the articles evidenced the association between nutritional status and the presence of chronic diseases, significantly compromising the quality of life of the elderly.
\end{abstract}

Keywords: Nutritional status; chronic disease; quality of life; middle aged; aged. 


\section{Introdução}

O envelhecimento da população é um fenômeno de amplitude mundial. A Organização das Naçóes Unidas (ONU) prevê que em 2025 existirão 1,2 bilhóes de pessoas com mais de 60 anos, sendo que os octogenários constituem o grupo etário de maior crescimento ${ }^{1}$.

$\mathrm{Na}$ década de 1960, o Brasil iniciou um processo de envelhecimento demográfico com particularidades que o destacaram na escala mundial. Poucos países desenvolvidos apresentam velocidade de envelhecimento populacional como o ocorrido no Brasil ${ }^{2}$.

Vários são os meios de melhorar a qualidade de vida daqueles que estáo em processo de envelhecimento, dentre os quais se incluem os programas de saúde. Esses programas implicam na promoçáo da saúde do idoso e devem ter a nutrição como uma área prioritária ${ }^{3}$. $\mathrm{O}$ estado nutricional demonstra o grau no qual as necessidades fisiológicas por nutrientes estão sendo atingidas para manutenção da composição e das funçôes adequadas do organismo ${ }^{4}$.

As variáveis nutricionais têm importantes implicaçóes no contexto do envelhecimento da população, visto que o controle de grande parte das doenças crônicas ou infecciosas e a prevenção de complicaçôes decorrentes das mesmas dependem do estado nutricional 5 .

A avaliação nutricional é aspecto importante nesse contexto, porque os idosos apresentam condiçóes peculiares, que comprometem o seu estado nutricional ${ }^{6}$. Alguns desses condicionantes ocorrem devido às alterações fisiológicas do próprio processo de envelhecer, enquanto outros são causados pelas doenças presentes pelas práticas ao longo da vida ${ }^{7}$.

Diversos estudos clínicos e de intervenção mostram a ligação direta entre qualidade da dieta e surgimento de doenças e agravos não transmissíveis, destacando a importância da alimentaçáo na saúde e no bem-estar ${ }^{3}$.

$\mathrm{O}$ estado nutricional interfere na capacidade funcional dos idosos e, por sua vez, pode ser influenciado por condiçóes mórbidas que comprometem a qualidade de vida desse grupo etário.

A manutenção de um estado nutricional adequado é fator primordial, pois de um lado encontra-se o baixo peso, que aumenta o risco de infecçóes e mortalidade e do outro o sobrepeso, que aumenta o risco de doenças crônicas não transmissíveis (DCNT) como hipertensão, cardiopatias, diabetes mellitus e hiperlipidemias ${ }^{8}$.

A elevada prevalência de doenças crônicas entre idosos brasileiros tem sido frequentemente referida, em especial as cardiovasculares, que constituem uma das principais causas de mortalidade, além de se associarem à incapacidade, dependência e perda de autonomia, com alto custo econômico e social? .

As doenças crônicas podem influenciar diretamente a autonomia e independência dos idosos e, consequentemente, gerar incapacidade levando a dependência funcional ${ }^{6}$. Quando estas dificuldades se manifestam ocorre o comprometimento da qualidade de vida.

O Grupo de Qualidade de Vida da OMS ${ }^{10}$ definiu qualidade de vida como a percepçáo do indivíduo de sua posiçáo na vida, no contexto cultural e no sistema de valores nos quais ele vive, em relação aos objetivos, às expectativas, aos padróes e às preocupaçóes.

O envelhecimento e a qualidade de vida são fenômenos multifacetados, que merecem atenção, destacando-se a necessidade de estudos que investiguem os comportamentos relacionados à saúde e ao estado nutricional dessa população. Dessa maneira, a revisão integrativa possibilita a discussão e análise dos resultados obtidos e a partir destes, é possível oferecer sugestôes para a realização de futuras pesquisas científicas ${ }^{3}$.

Este estudo tem como objetivo realizar uma revisão integrativa referente às alteraçóes do estado nutricional, ao aparecimento de doenças crônicas, assim como de suas inter-relaçóes e seu impacto na qualidade de vida dos idosos.

\section{Método}

Trata-se de uma revisão integrativa, realizada na Universidade São Judas Tadeu, em São Paulo.

Para a realização deste estudo, foram utilizados os seguintes descritores: "nutrition status", "60+ years", "chronic diseases", "quality of life", limitando os idiomas ao inglês e ao português, aos estudos realizados com seres humanos, aos textos na íntegra e aos temas compatíveis ao pesquisado neste trabalho, com limitadores temporais no período de publicação de 2009 até 2016, consultados nas bases de dados: Medline/PubMed (116 artigos), LILACS (9 artigos), SciElo (12 artigos) e Scopus (71 artigos).

A primeira seleção foi retirar a duplicidade nas bases de dados, de modo que restaram 113 artigos. Em seguida, ocorreu a escolha pelos títulos, que resultou em 87 publicaçôes selecionadas. Após a leitura dos resumos, foram excluídos 66 que não abordavam o tema compatível ao pesquisado. Restaram 21 artigos, que foram lidos na íntegra e, posteriormente, houve a exclusão daqueles que não atendiam ao objetivo. $\mathrm{O}$ estudo foi finalizado com a inclusão de 12 artigos (Figura 1)

\section{Resultados}

A apresentação dos resultados foi ilustrada no Quadro 1, com o objetivo de sintetizar as principais características metodológicas e os resultados dos artigos elegíveis. 
Figura 1: Diagrama do processo de obtenção do corpus

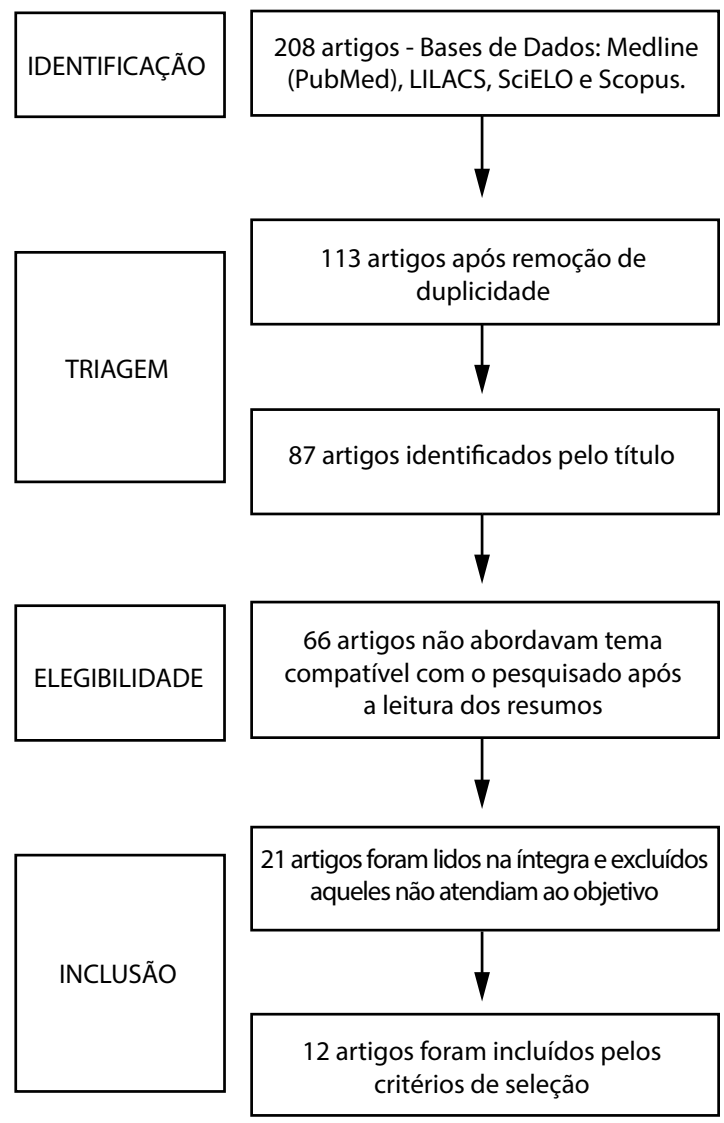

Quadro 1: Síntese dos estudos relacionados: a relação entre estado nutricional e presença de doenças crônicas e o impacto na qualidade de vida de idosos.

\begin{tabular}{|c|c|c|c|}
\hline Autor/Ano & $\begin{array}{l}\text { Característica da } \\
\text { populaçáo }\end{array}$ & Instrumentos de pesquisa & Resultados da pesquisa \\
\hline $\begin{array}{l}\text { Cintra, Oliveira e } \\
\text { Silva }^{11}\end{array}$ & $\begin{array}{l}\text { - } 197 \text { idosos ( } 70 \text { a } 80 \\
\text { anos) } \\
-75 \text { idosos voluntários } \\
\text { - } 122 \text { institucionalizados } \\
\text { Araraquara/SP }\end{array}$ & $\begin{array}{l}\text { - IMC } \\
\text { - CA } \\
\text { - Entrevista } \\
\text { - Consulta de prontuários de saúde }\end{array}$ & $\begin{array}{l}\text { Idosos institucionalizados: } 30 \% \text { baixo peso, } 40 \% \\
\text { sobrepeso. } \\
\text { Idosos voluntários: } 4 \% \text { baixo peso, } 60 \% \text { sobrepeso. } \\
\text { A ocorrência de hipertensão foi significativa nos dois } \\
\text { grupos. } \\
\text { A qualidade de vida dos idosos institucionalizados } \\
\text { obteve menor pontuação, provavelmente por apresen- } \\
\text { tarem distúrbio nutricional com maior frequência. }\end{array}$ \\
\hline Silva, Simôes e Leite ${ }^{12}$ & $\begin{array}{l}100 \text { idosos DM2 (60 a } 85 \\
\text { anos) } \\
\text { Araçatuba / SP }\end{array}$ & $\begin{array}{l}\text { - IMC } \\
\text { - CA } \\
\text { - Características sociodemográficas } \\
\text { - Estilo de vida } \\
\text { - História clínica e antecedentes fa- } \\
\text { miliares - Aplicação de questionário } \\
\text { validado } \\
\text { - Variáveis bioquímicas }\end{array}$ & $\begin{array}{l}42 \% \text { obesidade e } 40 \% \text { sobrepeso. } \\
\text { Doenças crônicas: } 54 \% \text { HAS, } 78 \% \text { diabetes. } \\
\text { O comprometimento do estado nutricional associado } \\
\text { ao aparecimento de doenças crônicas influenciou a } \\
\text { qualidade de vida. }\end{array}$ \\
\hline $\begin{array}{l}\text { Leite-Cavalcanti C, } \\
\text { Rodrigues-Gonçalves, } \\
\text { Rios-Asciutti, } \\
\text { Leite-Cavalcanti } \mathrm{A}^{13}\end{array}$ & $\begin{array}{l}117 \text { idosos ( } 60 \text { a } 87 \text { anos) } \\
\text { Joáo Pessoa / PB }\end{array}$ & $\begin{array}{l}\text { - IMC } \\
\text { - CC } \\
\text { - CQ } \\
\text { - Questionário: estilo de vida e fato- } \\
\text { res relacionados à saúde e DCNT } \\
\end{array}$ & $\begin{array}{l}97,5 \% \text { obesos, } \\
82 \% \text { apresentaram DCNT. } \\
\text { A elevada prevalência da obesidade associada à presença } \\
\text { de DCNT comprometeu significativamente a saúde } \\
\text { desses idosos e, consequentemente, a qualidade de vida. }\end{array}$ \\
\hline
\end{tabular}


Quadro 1: Continuação.

\begin{tabular}{|c|c|c|c|}
\hline Autor/Ano & $\begin{array}{l}\text { Característica da } \\
\text { populaçáo }\end{array}$ & Instrumentos de pesquisa & Resultados da pesquisa \\
\hline $\begin{array}{l}\text { Wachholz, Rodrigues } \\
\text { e Yamane }^{14}\end{array}$ & $\begin{array}{l}\text { - } 35 \text { idosos } \\
\text { institucionalizados } \\
\text { ( } 70 \text { a } 80 \text { anos) } \\
\text { Curitiba/PR }\end{array}$ & $\begin{array}{l}\text { - IMC } \\
\text { - WHOQOL-BREF } \\
\text { - Variáveis sociodemográficas }\end{array}$ & $\begin{array}{l}42 \% \text { baixo peso, } 14,3 \% \text { obesidade e } 8,6 \% \text { sobrepeso. } \\
\text { Idosos que apresentaram melhores médias nos domí- } \\
\text { nios que compóem o WHOQOL-BREF apresenta- } \\
\text { ram sobrepeso e menor idade. }\end{array}$ \\
\hline $\begin{array}{l}\text { Torres, Reis e } \\
\text { Fernandes }{ }^{15}\end{array}$ & $\begin{array}{l}-117 \text { idosos }(+ \text { de } 80 \\
\text { anos) } \\
\text { Jequié/BA }\end{array}$ & $\begin{array}{l}\text { - WHOQOL-OLD } \\
\text { - Índice de Barthel } \\
\text { - Dados sociodemográficos e condi- } \\
\text { çốes de saúde } \\
\text { - Apgar de família } \\
\text { - Questionário validado }\end{array}$ & $\begin{array}{l}\text { Não foi calculado IMC. } \\
23 \% \text { apresentaram hipertensão arterial. } \\
\text { O comprometimento da dinâmica familiar influenciou } \\
\text { o índice de qualidade de vida dos idosos dependentes, } \\
\text { quanto mais prejudicada a funcionalidade da família, } \\
\text { pior qualidade de vida. }\end{array}$ \\
\hline Alfonso-Rosa et al. ${ }^{16}$ & $\begin{array}{l}\text { - } 42 \text { idosos (60 a } 70 \text { anos) } \\
\text { Sevilha/Espanha }\end{array}$ & $\begin{array}{l}\text { - MAN } \\
\text { - European Arudety }\end{array}$ & $\begin{array}{l}25 \% \text { sobrepeso, } 15 \% \text { obesidade, } 38 \% \text { risco nutricio- } \\
\text { nal, } 12 \% \text { desnutriçáo. } \\
\text { A presença de DM tipo } 2 \text {, juntamente com o compro- } \\
\text { metimento do estado nutricional, influenciou de manei- } \\
\text { ra significativa a qualidade de vida dos idosos estudados. }\end{array}$ \\
\hline Artacho et al. ${ }^{17}$ & Jaen/Espanha & $\begin{array}{l}\text { - Variáveis sociodemográficas } \\
\text { - Questionário validado }\end{array}$ & $\begin{array}{l}\text { Segundo o WHOQOL-BREF, a qualidade de vida } \\
\text { dos idosos cronicamente doentes diminuiu com a } \\
\text { idade, independente dos parâmetros nutricionais. }\end{array}$ \\
\hline Donner Alves et al. ${ }^{18}$ & $\begin{array}{l}\text { - } 46 \text { idosos do sexo } \\
\text { masculino com insuficiência } \\
\text { cardíaca }(58 \text { a } 70 \text { anos }) \\
\text { Araras/SP }\end{array}$ & $\begin{array}{l}\text { - Variáveis sociodemográficas } \\
\text { - Questionário dietético: } \\
\text { recordatório } \\
\text { - IMC } \\
\text { - Exames bioquímicos }\end{array}$ & $\begin{array}{l}67 \% \text { sobrepeso, } 18 \% \text { obesidade e } 10 \% \text { risco } \\
\text { nutricional. } \\
\text { A orientaçáo nutricional para a diminuição de sódio } \\
\text { e gordura na dieta influenciou na diminuiçáo dos } \\
\text { riscos de IC e consequentemente melhorou o estilo e } \\
\text { a qualidade de vida. }\end{array}$ \\
\hline Rasheed e Woods ${ }^{19}$ & $\begin{array}{l}\text { (65 a } 99 \text { anos) } \\
\text { Inglaterra }\end{array}$ & $\begin{array}{l}\text { - IMC } \\
\text { - Inquérito } \\
\text { - EUROQOL (5d-3L) }\end{array}$ & $\begin{array}{l}13 \% \text { risco nutricional, } 47 \% \text { desnutrição e } 21 \% \text { de } \\
\text { baixo peso. } \\
\text { Hipertensão arterial: } 55 \% \text {. } \\
\text { Os idosos com idade avançada portadores de hiperten- } \\
\text { são e risco nutricional apresentam comprometimento } \\
\text { significativo na qualidade de vida. }\end{array}$ \\
\hline Jiménez et al. ${ }^{20}$ & $\begin{array}{l}\text { - } 83 \text { idosos (+80 anos) } \\
\text { Madrid/Espanha }\end{array}$ & $\begin{array}{l}\text { - MAN } \\
\text { - IMC } \\
\text { - Consumo alimentar dietético (24hrs) } \\
\text { - EUROQOL-5d (EQ-5d) }\end{array}$ & $\begin{array}{l}\text { Os idosos avaliados apresentaram fatores de risco para } \\
\text { DCNT. } \\
\text { 3\% dos homens apresentaram desnutriçáo e 6,7\% } \\
\text { risco nutricional. } \\
7,9 \% \text { das mulheres apresentaram desnutrição e } \\
\text { 37,7\% de risco nutricional. } \\
\text { As mulheres com presença de DCNT e risco } \\
\text { nutricional apresentam menor QV. }\end{array}$ \\
\hline Machado e Coelho ${ }^{21}$ & $\begin{array}{l}\text { - } 344 \text { idosos (+70 anos) } \\
\text { - Abrigos municipais } \\
\text { Rio de Janeiro/Brasil }\end{array}$ & $\begin{array}{l}\text { - MAN } \\
\text { - IMC } \\
\text { - CA } \\
\text { - Variáveis sociodemográficas, estado } \\
\text { de saúde e estilo de vida }\end{array}$ & $\begin{array}{l}8,3 \% \text { desnutrição, } 56,6 \% \text { risco nutricional e } 10 \% \\
\text { baixo peso. } \\
\text { Foram identificados fatores de risco para DCNT. } \\
\text { O índice de QV está associado à presença de DCNT } \\
\text { e ao estado nutricional: quanto maior o risco nutri- } \\
\text { cional, pior a qualidade de vida. }\end{array}$ \\
\hline Kostka et al. ${ }^{22}$ & $\begin{array}{l}-2.772 \text { idosos } \\
\text { (+60 anos), sendo } \\
1.003 \text { de comunida- } \\
\text { des urbanas, } 890 \text { de } \\
\text { comunidade rural e } 879 \\
\text { institucionalizados } \\
\text { Polônia }\end{array}$ & $\begin{array}{l}\text { - IMC } \\
\text { - MAN } \\
\text { - EUROQOL 5d }\end{array}$ & \\
\hline
\end{tabular}

Legenda:

IMC = Índice de massa corporal; $\mathrm{CA}=$ Circunferência abdominal; $\mathrm{CC}=$ Circunferência da cintura; $\mathrm{DCNT}=$ Doença crônica não transmissível; $\mathrm{MAN}=\mathrm{Mini}$ avaliação nutricional; $\mathrm{BP}=$ Baixo peso; $\mathrm{SP}=$ Sobrepeso; $\mathrm{RN}=$ Risco nutricional; $\mathrm{DN}=$ Desnutrição; $\mathrm{O}=$ Obesidade; $\mathrm{E}=$ Eutrófico; $\mathrm{EN}=\mathrm{Estado}$ nutricional; $\mathrm{ES}=$ Estado de saúde; $\mathrm{QV}=$ Qualidade de vida; $\mathrm{FRDCV}=$ Fator de risco para doenças crônicas vasculares. 


\section{Discussão}

A partir da análise dos estudos avaliados, observa-se que, apesar do envelhecimento ser um processo natural, as alteraçóes do estado nutricional e o aparecimento de DCNT podem comprometer definitivamente a qualidade de vida dos idosos.

A qualidade de vida reportada na terceira idade enfatiza a nutrição como fator determinante para que o idoso possa manter uma interação harmoniosa de variados fatores que moldam e diferenciam seu cotidiano, como saúde física e mental, satisfação no trabalho e nas relaçôes familiares, espiritualidade e longevidade ${ }^{6}$.

Os distúrbios nutricionais apresentam-se em forma de desnutriçáo, sobrepeso e obesidade. De acordo com LeiteCavalcanti et al. ${ }^{13}$, a maioria dos idosos com excesso de peso e com menos idade apresenta maior satisfação com sua vida, com uma melhor percepçáo dos aspectos que influenciam a qualidade de vida. Por outro lado, apresenta maior risco para o desenvolvimento de DCNT, principalmente doenças cardiovasculares e diabetes mellitus.

Nutrição e saúde estão intimamente relacionadas e remetem à noção de um estilo de vida saudável, que inclui a possibilidade de manter-se ativo e independente, de forma a garantir uma alimentaçáo satisfatória, que contribua para a manutençáo da qualidade de vida, atrelada a fatores emocionais e sociais ${ }^{8}$.

Os idosos desnutridos apresentam-se mais depressivos, sem expectativas. As alteraçóes do estado nutricional são decorrentes, frequentemente, da falta de apetite e alteração do paladar. Esses idosos apresentam redução na qualidade de vida e elevados índices de mortalidade e de susceptibilidade às infecções, bem como alta incidência de fraturas, problemas respiratórios e cardíacos ${ }^{6}$.

Uma das dificuldades ao diagnosticar esse distúrbio nutricional é o fato de ele ser facilmente confundido com as modificações orgânicas naturais do processo de envelhecimento, de forma que as intervenções, muitas vezes, apresentam-se inadequadas e menos potentes do que podem ser, contribuindo para o agravamento de manifestações clínicas associadas a inúmeras doenças crônicas ${ }^{4}$.

Cintra et al. ${ }^{11}$ avaliaram a qualidade de vida de idosos institucionalizados e não institucionalizados e concluíram que os institucionalizados apresentam, além de distúrbios nutricionais, alteraçóes no estado emocional causados pela ausência da família, que interfere nas relaçóes sociais, nos hábitos alimentares e na qualidade de vida desses indivíduos. O conforto domiciliar pode ser interpretado simplesmente como situação que produz bem-estar.

Artacho et al. ${ }^{13}$ mostraram que os idosos com idades avançadas apresentaram maior susceptibilidade ao aparecimento de DCNT, principalmente doenças cardiovasculares, com comprometimento do estado nutricional e consequente alteração da qualidade de vida associada a vários fatores que impactam negativamente a saúde em geral.

O estudo de Alves et al. ${ }^{17}$ mostrou que a constatação da doença impóe modificações em todas as situaçôes do cotidiano, levando o idoso a buscar novos hábitos de vida. Essas mudanças são necessárias, e os resultados dessas alteraçóes na qualidade de vida, muitas vezes, contribuem para um aprendizado mais eficaz, com o objetivo de assegurar-lhes mais independência e saúde.

Assim, o conhecimento das características e das transformações por que passam os idosos, sejam elas sistêmicas, fisiológicas ou anatômicas, além dos fatores relacionados à saúde que resultam do estilo de vida, assume um papel relevante na assistência ao idoso.

Observa-se nos estudos avaliados a falta de padronização em relação aos instrumentos utilizados para diagnóstico nutricional e para avaliação de qualidade de vida, dificultando as análises e a comparação dos resultados.

\section{Conclusão}

Os estudos avaliados mostraram que idosos com baixo peso ou com obesidade apresentaram associação com pior qualidade de vida e menor grau de satisfação. Também foi observado que alteraçóes nutricionais associadas à presença de DCNT afetam diretamente a qualidade de vida dos idosos.

Os autores citados parecem concordar em relaçâo à necessidade de ampliar estratégias específicas de promoção à saúde do idoso e prevenção de doenças. Dessa forma, a identificação dos determinantes do comportamento alimentar e das condiçóes de saúde dos idosos são fatores importantes para o estabelecimento de políticas públicas destinadas à melhoria da qualidade de vida e promoção de um envelhecimento mais saudável.

\section{Referências}

1. Sousa L, Galante H, Figueiredo D. Qualidade de vida e bem-estar dos idosos: um estudo exploratório na população portuguesa. Rev Saude Publica. 2003;37(3):364-71.

2. Wong LLR, Carvalho JA. O rápido processo de envelhecimento populacional no Brasil: sérios desafios para as políticas públicas. R bras Est Pop. 2006;23(1):5-26.

3. Cervato AM, Derntl AM, Latorre MRDO, Marucci MFN. Educação nutricional para adultos e idosos: uma experiência positiva em Universidade Aberta para a Terceira Idade. Rev Nutr. 2005;18(1):41-52. 
4. Tavares EL, Anjos LA. Perfil antropométrico da população idosa brasileira. Resultados da Pesquisa Nacional sobre Saúde e Nutrição. Cad Saúde Pública. 1999;15(4):759-68.

5. World Health Organization. Physical status: the use and interpretation of anthropometry. Geneva: WHO; 1995. (Technical Report Series, 854).

6. Sampaio LR. Avaliação nutricional e envelhecimento. Rev Nutr. 2004;17(4):507-14.

7. Amado TCF, Arruda IKG, Ferreira RAR. Aspectos alimentares, nutricionais e de saúde de idosas atendidas no Núcleo de Atenção ao Idoso - NAI, Recife/2005. ALAN. 2007;57(4):366-72.

8. Bueno JM, Martino HSD, Fernandes MFS, Costa LS, Silva RR. Avaliação nutricional e prevalência de doenças crônicas não transmissíveis em idosos pertencentes a um programa assistencial. Ciên Saúde Col. 2008;13(4):1237-46.

9. Campos MAG, Pedrosa ERP, Lamounier JA, Colosimo EA, Abrantes MM. Estado nutricional e fatores associados em idosos. Rev Assoc Med Bras. 2006;52(4):214-21.

10. World Health Organization. The World Health Organization Quality of Life Assessment (WHOQOL): development and general psychometric properties. Soc Sci Med. 1998;46(12):1569-85.

11. Cintra RMGC, Oliveira D, Silva LMG. Estado nutricional e ocorrência de hipertensão arterial e de diabetes em idosos residentes e não residentes em instituiçôes geriátricas. Alim Nutr. 2012;23(4):567-75.

12. Silva RCP, Simóes MJS, Leite AA. Fatores de risco para doenças cardiovasculares em idosos com diabetes mellitus tipo 2. Rev Ciênc Farm Básica Apl. 2007;28(1):113-21.

13. Leite-Cavalcanti C, Rodrigues-Gonçalves MC, RiosAsciutti LS, Leite-Cavalcanti A. Prevalência de doenças crônicas e estado nutricional em um grupo de idosos brasileiros. Rev salude publica. 2009;11(6):865-77.

14. Walchholz PA, Rodrigues SC, Yamane R. Estado nutricional e a qualidade de vida em homens idosos vivendo em instituição de longa permanência em Curitiba, PR. Rev Bras Geriatr Gerontol. 2011;14(4):625-35.

15. Torres GV, Reis LA, Reis LA, Fernandes MH. Qualidade de vida e fatores associados em idosos dependentes em uma cidade do interior do Nordeste. J Bras Psiquiatr. 2009;58(1):39-44.

16. Alfonso-Rosa RM, Del Pozo-Cruz B, Del Pozo-Cruz J, Sańudo B, Rogers ME. The relationship between nutritional status, functional capacity and health-related quality of life in older adults with type 2 diabetes: a pilot explanatory study. J Nutr Health Aging. 2013;17(4):315-21.

17. Artacho R, Lujano C, Sanchez-Vico AB, Vargas Sánchez C, González Calvo J, Bouzas PR, et al. Nutritional status in chronically-ill elderly patients. Is it related to quality of life? J Nutr Health Aging. 2014;18(2):192-7.

18. Donner Alves F, Correa Souza G, Brunetto S, Schweigert Perry ID, Biolo A. Nutritional orientation, knowledge and quality of diet in heart failure: randomized clinical trial. Nutr Hosp. 2012;27(2):441-8.

19. Rasheed S, Woods RT. An investigation into the association between nutritional status and quality of life in older people admitted to hospital. J Hum Nutr Diet. 2014;27(2):142-51.

20. Jiménez-Redondo S, Beltrán de Miguel B, Gavidia Banegas J, Guzmán Mercedes L, Gómez-Pavón J, Cuadrado Vives C. Influence of nutritional status on health-related quality of life of non-institutionalized older people. J Nutr Health Aging. 2014;18(4):359-64.

21. Machado RSP, Coelho MASC. Risk of malnutrition among Brazilian institutionalized elderly: a study with the Mini Nutritional Assessment (MNA) questionnaire. J Nutr Health Aging. 2011;15(7):532-5.

22. Kostka J, Borowiak E, Kostka T. Nutritional status and quality of life in diferente populations of older people in Poland. Eur J Clin Nutr. 2014;68(11):1210-5.

\section{Como citar este artigo:}

Lira S, Goulart RM, Alonso AC. A relação entre estado nutricional e presença de doenças crônicas e seu impacto na qualidade de vida de idosos: revisão integrativa. Rev. Aten. Saúde. 2017;15(53):81-86. 